\title{
Arzt-Patienten-Beziehung - Defizite in der Weiterbildung zum Psychiater?
}

\section{Daniel Bielinski}

Vizepräsident der Schweizerischen Gesellschaft für Psychiatrie und Psychotherapie SGPP
Korrepondenz:

Dr. med. Daniel Bielinski

Psychiatrische Dienste Aargau AG Zürcherstrasse 241

$\mathrm{CH}-5210$ Windisch

bielinski[at]bluewin.ch
An einem Workshop zum Thema «Arzt-Patienten-Beziehung» wurde ich mit der Tatsache konfrontiert, dass die Patienten sich in der Beziehung zu ihrem Arzt nicht wohl fühlen. Die Beziehungsgestaltung ist ungenügend, die Patienten fühlen sich nicht abgeholt, können sich nicht frei äussern, haben den Eindruck, es gäbe zu wenig Zeit, zu wenig Fokussierung auf ihre Probleme. Die dazu präsentierten Fallbeispiele untermauern den Notstand. Pharmazeutische Firmen, die meistens recht frühzeitig Trends im Gesundheitswesen erkennen, preisen Kommunikationsworkshops zur Gestaltung der Arzt-Patienten-Beziehung an.

Was ist passiert? Wir alle haben gelernt, dass die Arzt-Patienten-Beziehung das tragende Fundament ist, worauf dann im Fach der Psychiatrie der biopsychosoziale Ansatz in der Behandlung aufbaut. Nun bekommen wir das ziemlich ernüchternde Feedback, dass das Fundament nicht trägt. Beim Hausbau würde man sich beim Architekten und beim Statiker beschweren, würde anregen, dass dringend nachgebessert wird, im Wissen, dass ohne das Fundament das Haus nicht nachhaltig gebaut ist. Doch wir sind Architekten und Statiker im Bereich der Aus-, Weiterund Fortbildung? Wer selektioniert Menschen, die offenbar in der Beziehungsgestaltung Defizite aufweisen, die sie dann während des Studiums und der Weiterbildung nicht mehr aufholen können? Oder ist es ein gesellschaftlicher Trend? Oder sind es einfach die Patienten, die anspruchsvoller geworden sind?

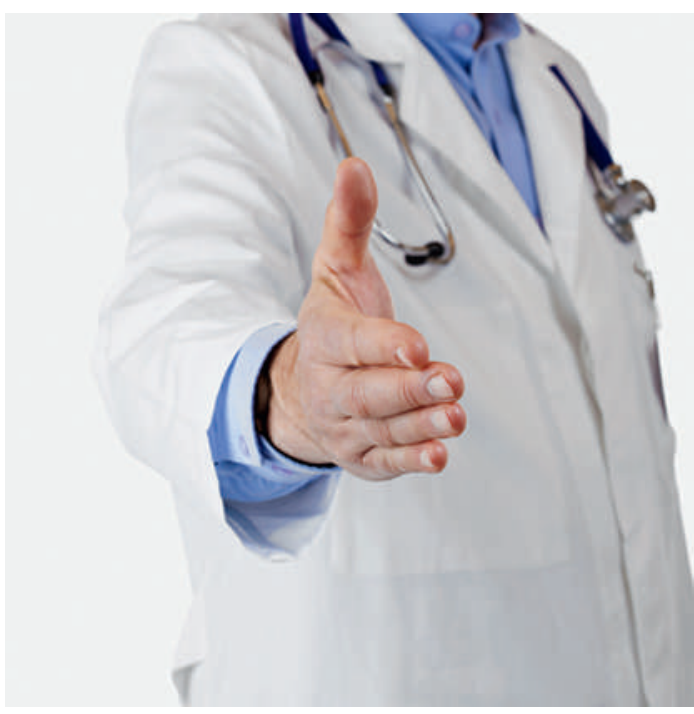

Ein Warnsignal: Viele Patienten fühlen sich in der Beziehung zum Arzt nicht wohl.
Aus meiner Sicht spielen viele Faktoren eine Rolle: Ich denke, dass der Numerus clausus nach wie vor darauf ausgerichtet ist, solche Studenten zum Medizinstudium zuzulassen, die den Anforderungen des Studiums am besten genügen. Eine Selektion in Richtung Eignung im Bereich der Beziehungsgestaltung, der Kommunikation, des Einfühlungsvermögens usw. findet nicht statt. Entsprechend mag es nicht ganz erstaunen, dass Ärzte in die Weiterbildungsphase aufsteigen, die vielleicht von ihrer Persönlichkeit her nicht primär für ihren späteren Beruf geeignet sind. Die Anforderungen in der Ausbildung zum Mediziner wurden massiv hochgeschraubt, als Feigenblatt für die Grundlage des späteren Berufes wird die psychosoziale Medizin gelehrt.

\section{Eine Selektion in Richtung Eignung im Bereich der Beziehungs- gestaltung findet nicht statt.}

Gerade in der Weiterbildung zum Psychiater sollten die Beziehungsaspekte einen ganz zentralen Platz einnehmen. Es kann doch nicht sein, dass in dem Beruf, wo die Passung zwischen Patient und Therapeut eine so zentrale Rolle spielt, die Patienten sich zum grösseren Teil nicht wohl fühlen, mit ihren Problemen und Sorgen nicht abgeholt werden.

Wo ist der Beitrag der direkten Weiterbildner, der verschiedenen Psychotherapieausbildungen? Die Fokussierung auf «bio» im biopsychosozialen Ansatz ist aus meiner Sicht mitverantwortlich für diese negative Entwicklung. Es gibt Psychiater, die regeln mit ihren depressiven Patienten innerhalb von 10 Minuten die Medikamenteneinnahme und verweisen sie dann zum Gespräch an die Psychologen. Dieses Aufteilen des integrativen Ansatzes ist sicher nicht dazu geeignet, Vertrauen in die Arzt-Patienten-Beziehung aufzubauen. Gleiches kann auch über die delegierte Psychotherapie gesagt werden.

So weit genug gejammert, die Missstände sind evident, wie nun weiter? Aus meiner Sicht gilt es, zunächst im Auswahlverfahren der zukünftigen Mediziner dem Aspekt der Arzt-Patienten-Beziehung mehr Raum zu geben. In anderen Ländern ist das Selektionsverfahren auf die entsprechenden Eigenschaften der Medizinstudenten ausgerichtet. Nur wir hier in der 
Schweiz lassen es mit stumpfen Eignungstests bewenden, finden es nicht notwendig, ein Gespräch mit dem Kandidaten zu führen. Die Ausbildung, mit ihrem vollgestopften Programm, hat es offenbar unterlassen, den zentralen Aspekt des medizinischen Handels so ins Zentrum zu rücken, dass das Fundament jeglichen ärztlichen Handelns solide und nachhaltig aufgebaut wird. miert ihren eigenen Ansatz, ohne sich dabei auf ein für alle Psychotherapieschulen gemeinsames Grundgerüst in der Psychotherapie der Beziehung ArztPatient abstützen zu können. Diesen Part müssen wir mangels fehlender Koordination der Psychotherapieschulen, Psychotherapieinstitute nun selbst übernehmen. Es ist unsere Aufgabe, dass der Berufsstand des Psychiaters nicht durch eine fatale Fehlentwicklung

\section{Nur wir in der Schweiz lassen es mit stumpfen Eignungstests bewenden, finden ein Gespräch mit dem Kandidaten nicht notwendig.}

In der Weiterbildung muss dem psychiatrischen Handwerk wieder mehr Platz eingeräumt werden. Das stumpfe Abfragen von Skalen, diagnostischen Katalogen verführt die jungen Mediziner dazu, ohne Beziehungsaufbau den Patienten möglichst rasch und messbar in ein diagnostisches Schema einzuzwängen. Denn, ist die Diagnose einmal gestellt, gibt es Behandlungsalgorithmen, die mechanisch abgespult werden können, ohne dass ein vertieftes Auseinandersetzen mit dem Menschen, den Patienten, stattfinden muss. Das Pendel hat hier in die gegenteilige Richtung voll ausgeschlagen. Hatten wir früher einen deutlichen Mangel an objektivierbaren Psychostaten, diagnostischen Kriterien und Standards in den Therapien, ist heute alles standardisiert, mechanisch ohne eben das Zentrale - nämlich die Arzt-Patienten-Beziehung - im Auge zu behalten. Aus meiner Sicht muss die zukünftige Weiterbildung diesen Defiziten zu Leibe rücken. Dies ist eine standespolitische Aufgabe. Auch hier sind die Zeichen schon lange spürbar, dass etwas nicht stimmt.

Es kann doch einfach nicht sein, dass wir Kollegen am Ende ihrer Weiterbildungszeit bei der Diskussion der Facharztprüfungen sehr stark ans Herz legen müssen, sich doch auch über die Beziehungsqualität zum Patienten zu äussern. Wir haben zwar die formalen Kriterien angepasst. Wir sagen, es interessiert uns, wie es um die Arzt-Patienten-Beziehung steht, wir haben aber das Problem dahinter nicht in den Griff bekommen.

Aus meiner Sicht ist es auch etwas das Problem, der verschiedenen Psychotherapieschulen. Jede opti- in Misskredit gerät. Der Arztberuf, der die Arztbeziehung ins Zentrum stellen sollte, sieht sich nun durch neuere Untersuchungen mit der Tatsache konfrontiert, dass wir oftmals an den Patienten «vorbeitherapieren».

Natürlich gibt es Begleiterscheinungen, die diese Entwicklung fördern, ich denke an die zunehmende Bürokratisierung, die mit dafür verantwortlich ist, dass heute ein Assistenzarzt in der Weiterbildung nur mehr 50\% seiner Zeit am Patienten verbringt. Gleiches gilt für den zuständigen Weiterbildungsoberarzt, der immer weniger Gelegenheit hat, zusammen mit dem Weiterbildungskandidaten gemeinsam Patientengespräch durchzuführen. Natürlich liegt es auch daran, dass die grossen Vorbilder, die den Weiterbildungskandidaten Orientierung geben, am Aussterben sind. $\mathrm{Zu}$ stark werden wir auch in den Führungsetagen der Spitäler zu Technokraten degradiert, die immer weniger profiliert für das Handwerk der Psychiatrie eintreten können.

Zusammenfassend stelle ich fest, dass wir in der Aus-, Weiter- und Fortbildung grosse Mängel, bezogen auf die Gestaltung der Arzt-Patienten-Beziehung, konstatierten. Die Analyse der Ursachen ist breit. Ebenso müssen wir die Lösungsvorschläge, das ganze Spektrum von der Selektion fürs Medizinstudium bis Anpassung der Weiter- und Fortbildung-Curricula anpassen. Persönlich betrachte ich es als zen trale standespolitische Aufgabe, neben den gewerkschaftlichen Aspekten auch die Identität unseres Berufs wieder pro-aktiv dorthin zu entwickeln, wo wir das Optimale für unsere Patienten erreichen wollen. 